

AIAA 2000-4848

Multidisciplinary Techniques and Novel Aircraft Control Systems

Sharon L. Padula, James L. Rogers, and David L. Raney NASA Langley Research Center

Hampton, VA 23681

$8^{\text {th }}$ AIAANASA/USAF/ISSMO Symposium on Multidisciplinary Analysis and Optimization September 6-8, 2000 / Long Beach, CA 


\title{
MULTIDISCIPLINARY TECHNIQUES AND NOVEL AIRCRAFT CONTROL SYSTEMS
}

\author{
Sharon L. Padula., James L. Rogers, ${ }^{\dagger}$ and David L. Raney ${ }^{\ddagger}$ \\ NASA I angley Research Center \\ Hampton, VA 23681
}

\begin{abstract}
The Aircraft Morphing Program at NASA Langley Research Center explores opportunities to improve airframe designs with smart technologies. Two elements of this basic research program are multidisciplinary design optimization (MDO) and advanced flow control. This paper describes examples where MDO techniques such as sensitivity analysis, automatic differentiation, and genetic algorithms contribute to the design of novel control systems. In the test case, the design and use of distributed shapechange devices to provide low-rate maneuvering capability for a tailless aircraft is considered. The ability of MDO to add value to control system development is illustrated using results from several years of research funded by the Aircraft Morphing Program.
\end{abstract}

\section{Introduction}

Researchers who specialize in multidisciplinary design optimization (MDO) and those who specialize in optimal control have a natural affinity, since all use mathematical optimization techniques. Yet. few examples of MDO research including flight control as one of the disciplines exist. This apparent contradiction stems from the traditional process of designing aircraft flight control systems. Traditional aircraft conceptual design (see for example Ref. 1) assumes a small number of conventional control devices: ailerons (roll effectors), elevator (pitch effector), and rudder (yaw effector). The size and location of these devices can be estimated by using historical databases for component weight and control effectiveness information. The detailed control law design is postponed until the aircraft configuration is frozen and until precise control moments are measured or predicted.

The Aircraft Morphing Program envisions new types of control devices, such as inflatable bladders or oscillatory jets distributed over the wing surface, which achieve control by changing the real or virtual shape of the wing." These novel control systems require an equally revolutionary control design process. This paper suggests that MDO techniques, such as automatic differentiation for calculating sensitivities and genetic algorithm (GA) optimization procedures, are essential components of that new process.

\section{Control System Design Process}

The proposed control system design process is illustrated by application to a tailless fighter aircraft concept. The numerous control devices (effector arrays) were modeled as generic shape-change devices that respond immediately to commands from the

\footnotetext{
"Senior research scientist, Multidisciplinary Optimization Branch, MS 159. Senior Member AIAA.

* Senior computer scientist, Multidisciplinary Optimization Branch, MS 159.

‡ Senior research scientist, Dynamics and Control Branch. MS 406, Member AIAA.
}

Copyright (C) 2000 by the American Institute of Aeronautics and Astronautics, Inc. No copyright is asserted in the United States under Title 17, U.S. Code. The U.S. Government has a royalty-free license to exercise all rights under the copyright claimed herein for Governmental Purposes. All other rights are reserved by the copyright owner. 
controller. This kind of simplification was justified because the aim of the paper is to demonstrate $\mathrm{MDO}$ techniques.

Figure I illustrates the proposed control system design process. In Fig. 1, the arrows indicate the flow of data and the boxes represent steps in the design process. For example, the first step is to develop the vehicle concept and the final step is to develop and test the control law. The arrow which connects the final box to the initial box indicates that unsatisfactory results from the control law design may necessitate changes in the vehicle concept. In Fig. 1, the boxes with a dark border represent steps where MDO techniques can add value. The boxes with a light border represent steps where engineering judgement is especially important. Each step in the process is described in this section.

\section{Development of Vehicle Concept}

The first step in this multidisciplinary control system design process was to create a computational fluid dynamics (CFD) model of the vehicle and the control devices. In the present research, generic shape-change devices controlled a representative aircraft configuration called ICE (Innovative Control Effectors), created by Lockheed Martin $\$$. The ICE design, described in Refs. 3 and 4 and shown in Fig. 2. was used under a cooperative agreement with Lockheed Martin. The effectors were shape-change devices modeled as bumps on the surface of the wing. For the current proof-of-concept studies, existing grid points were deformed in the direction of the surface normal to represent potential shape-change devices. The CFD model of the ICE configuration is evaluated by an aerodynamic panel code called PMARC. ${ }^{5}$

\section{Prediction of Control Moments}

The second step was to predict the sensitivities of the control moments to a shape change at each grid point of the CFD model. The sensitivities were obtained by automatic differentiation of PMARC with the ADIFOR code generation tool." The sensitivities required were $\left(\frac{\partial C_{l}}{\partial h}, \frac{\partial C_{m}}{\partial h}, \frac{\partial C_{n}}{\partial h}\right)$, the partial derivatives of roll $\left(C_{l}\right)$, pitch $\left(C_{m}\right)$, and yaw $\left(C_{n}\right)$ moments with respect to a displacement $h$. The details of calculating a surface normal at every grid point and applying a change in

\footnotetext{
$\$$ The use of trademarks or names of manufacturers in this report is for accurate reporting and does not constitute an official endorsement, either expressed or implied, of such products or manufacturers by the National Aeronautics and Space Administration.
}

height $h$ along that normal are described by Park in Ref. 7. For the present ICE configuration model, these calculations required the derivatives of 3 output quantities with respect to 1394 input quantities. Calculating such a large number of derivatives was practical because of the adjoint option in version 3.0 of the ADIFOR software. ${ }^{\gamma}$ Park estimates that he spent a total of about one week modifying the PMARC code and applying ADIFOR-3.0. Calculating the sensitivities required about one hour of CPll time on a high speed engineering workstation.

Given a $3 \times 1394$ matrix of partial derivatives for grid locations on the right wing, the partial derivatives for corresponding locations on the left wing were constructed by assuming they had same magnitudes but the roll and yaw derivatives had opposite sign. Given this matrix of all partial derivatives, the moments $m$ resulting from activation of any set of effectors was estimated with a matrix multiplication:

$$
m=[B] \mu_{c m d}
$$

where $u_{c m i}$ is an $n$-vector of device heights $h$ and the matrix $B$ is constructed by selecting the $n$ columns associated with those devices. Note that this method of estimating moments implies linear superposition. which neglects control effector interactions. On the other hand, if $m_{\text {ctud }}$ represents a vector of desired roll, pitch, and yaw moments, then $u_{\text {rmd }}$ can be calculated by using the pseudo-inverse allocation method discussed in Ref. 9:

$$
u_{c m d}=B^{T}\left[B B^{T}\right]^{-1} m_{c m d}
$$

where $T$ denotes the matrix transpose. As in Ref. 9 , heights were restricted to positive values less than some maximum achievable device height. Therefore, if any element of $u_{i m t}$ was negative that element could be set to zero, and the corresponding element on the opposite wing could be increased by the same value. Then the resulting vector was normalized so that the maximum element equaled the maximum achievable device height. Finally, Eq. (1) was evaluated and the target moments $m_{\text {om }}$ were compared with the achievable moments $m$.

\section{Definition of Effector Array Candidates}

Fquations (1) and (2) were used to estimate the control moments produced by an array of effectors on the IC $E$ vehicle. An interactive design tool was created to let a researcher quickly build up and analyze potential locations (i. e., effector arrays) by selecting grid points 
and assigning device heights. This interactive tool, which uses MATLAB software developed by The MathWorks, Inc., helps the control law designer to determine good potential locations so that each device can produce the forces and moments required to maneuver the vehicle. Figure 3 illustrates the graphical user interface for the MATLAB-based effector array design tool. The wing planform on the left side of the display presents the CFD grid. The three contour plots on the right side of the display present the sensitivity data produced by step 2 . The top contour plot indicates roll moments on the top and bottom wing surfaces, the middle plot indicates pitching moments, and the bottom plot indicates yaw moments.

Once an array of effectors were defined by specifying locations and heights, the designer obtained a preliminary prediction of the arrays effectiveness based on Eqs. ( 1 ) and (2) and ADIFOR sensitivities. Next, he generated a perturbed geometry grid that included the deployed effector array. This geometry file could then be reevaluated with the PMARC aerodynamic analysis program. New sensitivity derivatives were calculated based on the perturbed PMARC: results. These PMARC-based sensitivities could be used with Eqs. (1) and (2) to further assess the effectiveness of the array. For the present study, perturbed device heights were less than or equal to 0.2 feet.

Figure 4 displays a $17 \times 41$ grid of candidate device locations on the right upper wing. Circles indicate one selected effector array. This array contains 28 devices. A right and left pair of these wing tip arrays can provide the estimated roll, pitch, and yaw moments shown in the first column of Table 1. Reanalysis of these same devices with a deformed grid in PMARC gives slightly modified estimates shown in the second column of Table 1. In this way, $\mathbf{3 4}$ different effector arrays were selected and evaluated. The two methods of estimating moments were quite consistent; in general the ADIFOR estimates were smaller than the PMARC estimates. Therefore, reliance on ADIFOR estimates for optimization should produce a conservative design.

Table 1. Control Moment Estimates Compared with PMARC Reanalysis Results.

\begin{tabular}{|l|l|l|}
\hline Moment & ADIFOR & PMARC \\
\hline Roll & $-1 .+4 \mathrm{E}-04$ & $-1.56 \mathrm{E}-(04$ \\
\hline Pitch & $-2.02 \mathrm{E}-04$ & $-2.20 \mathrm{E}-(04$ \\
\hline Yaw & $-0.22 \mathrm{E}-04$ & $-0.28 \mathrm{E}-04$ \\
\hline
\end{tabular}

The PMARC estimates for the effector arrays are plotted in Fig. 5. Estimates based on Eqs. (1) and (2), with sensitivity information generated by ADIFOR, would create a very similar plot. The candidate effector arrays were located in 7 regions: on the upper and lower leading edge (I.E), on upper and lower trailing edge (TE), on upper and lower wing tips, and on the upper surface near the middle of the wing. The arrays were not necessarily disjoint. In fact, some devices were members of several effector arrays.

The ellipses in Fig. 5 indicate a suite of four effector arrays that were studied in Ref. 9. This suite was used in a six-degree-of-freedom dynamic simulation to investigate the unaugmented and augmented aircraft dynamics. Results of that simulation are reported in Ref. 9 and indicate that a 10-degree-per-second roll rate is the maximum achievable with this suite of effectors. Those results suggest that this particular suite could be valuable for mild maneuvering or could be used in an autopilot to keep the wings level but could not take the place of conventional control surfaces.

\section{Selection of Optimal Effector Arrays}

The manually selected effector suite studied in Ref. 9 required 82 individual devices and did not completely meet the goals set by the designer. Given enough time and good intuition, the designer might have selected other effector suites with better characteristics. Alternately, the initial exploration for candidate effector suites can be accomplished with discrete optimization techniques. For example, Ref. 10 contains a literature survey of actuator placement research that indicates gool results for a wide range of applications.

With the MATLAB-based tool, the designer can define a large number of arrays and then can predict their control moments by using the PMARC analysis code. However, selecting the best set of arrays from this potential pool is a combinatorial problem that can be solved by using MDO techniques. The current study selected a GA-based optimization approach. The goal of the optimization was to reduce the number of devices required and to satisfy control effectiveness criteria. The GA is not suggested as a replacement for the designer, but as a tool for screening potential effector arrays and thus allowing the designer to consider only the most promising.

A multilevel GA, used to select control device locations, is described in Ref. 11. The goal of the GA is to select the minimum set of devices that can provide the required uncoupled control moments (e. g., provide sufficient pitching moment without adverse roll or yaw). That GA technique was tuned and validated with a simplified wing model. ${ }^{11.12}$ 
In the current study, the GA described in Ref. 11 was adapted to select effector arrays on the ICE model. Again, the objective was to find the minimum number of devices required to provide uncoupled roll, pitch, and yaw moments. Each member in the GA population represented one possible effector suite. Each individual was evaluated three times to determine roll, pitch, and yaw moments and to compare these achievable values with the target values. Failure to meet any of these targets caused a penalty to be added to the fitness (i. e., objective) function.

In Ref. 11, the penalty has a fixed size. After some experimentation, a step-linear penalty was determined to work better. Thus the fitness function, J, can be written as follows:

$$
J=n+\left[w_{l}\left(\frac{C_{l}^{*}}{C_{l}}\right)+w_{m}\left(\frac{C_{m}^{*}}{C_{m}}\right)+w_{n}\left(\frac{C_{n}^{*}}{C_{n}}\right)\right]
$$

where $n$ is the number of devices, $w_{i}$ are the minimum penalties, $C_{i}$ are the moments and $C_{i}{ }^{*}$ are the targets. For cases reported in this paper, $w_{i}=150$ if the moments are less than the targets (e. g., if $C_{i}<C_{i}^{*}$ ) and $w_{i}=0$ otherwise. The number 150 is an appropriate size for the minimum penalty because it has the same order of magnitude as $n$ judging from the number of devices in the manually-selected suite. Thus, the GA will be encouraged to drive all the penalty terms to zero by choosing a sufficient number of devices.

For the current study, the GA was developed and tested in two phases. During phase I, the 34 effector arrays selected by Raney were defined as the set of possible arrays. The GA was allowed to select up to 7 arrays from the original 34 . The effectiveness estimate for each suite of effector arrays was based on the PMARC. reanalysis data used in Ref. 9. The target values (see Eq. 3) were set to $C_{1}^{*}=6.0 \mathrm{E}-04, C_{m}{ }^{*}=5.0 \mathrm{E}-04$, and $C_{n}^{*}=3.0 \mathrm{E}-04$. During phase II, a set of 349 individual devices could be selected independently. The effectiveness estimate for each suite of devices was hased on the ADIFOR data. The target values for phase II are set to $C_{1}^{*}=5.0 \mathrm{E}-04, C_{m} *=5.0 \mathrm{E}-04$, and $C_{n}{ }^{*}=1.0 \mathrm{E}-04$; the targets were reduced because the estimates based on ADIFOR data consistently underestimated roll and yaw.

Obviously, phase I was a much smaller combinatorial problem, but it had its own complications. For example, some of the arrays overlapped and so not all possible combinations were allowable. To circumvent this problem, the GA was allowed to pick at most one array from each of the 7 regions shown in Fig. 5. The GA implementation would be simplified if each region had the same number of arrays. So, because the upper TE region contained 8 arrays, arrays in the other regions were duplicated until each region contained 8 arrays. In this way, the string length for the phase I GA was set to 7 and each digit in the string could have a value between 0 and 8 . Thus, the number of possible combinations was $9^{7}$ (approximately 4,800,000). although not all of these combinations represented unique effector suites. The duplication of effector arrays should not have had a significant impact on the convergence of the GA. Although, duplication does produce many members of the population with the same fitness value, a $\mathrm{GA}$ is especially well suited for optimization problems with this characteristic.

The GA population size for phase I was 200. An initial population of members was produced randomly and their fitness evaluated. Successive generations were produced by the GA operations of tournament selection, uniform crossover, and mutation, with a mutation rate of $5 \%$. The maximum number of generations was set to 300 . A single execution consisting of 300 generations of the GA procedure requires about one hour on a engineering workstation. For complete descriptions of GA techniques and definitions of GA parameters, see Ref. 13.

Typical results of the GA are shown in Fig. 6. Notice that the scales on each figure are different in order to emphasize several points about the convergence history. Figure 6a shows the value of the fitness function averaged over all population members in each generation. The maximum and minimum fitness is also plotted. Figure 6a shows clearly that the population maintained adequate diversity for 300 generations. Figure $6 \mathrm{~b}$ shows the average and minimum fitness for the first $\mathbf{2 0}$ generations. Notice that the average fitness reduced dramatically over this interval and approached the value of 150 . This trend suggests that all members of the population were converging towards designs whose achievable moments were close to or better than the target values. Figure $6 c$ shows the best fitness ever calculated as a function of generation number. Figure $6 c$ indicates the original population contained at least one member with a fitness less than 150, suggesting that some members met all the performance targets. Notice also that the best individual had 96 devices and was found before generation 20 .

Even though 300 generations and about 60,000 different effector suites (i. e., about $1.25 \%$ of all possible combinations) were evaluated by the GA, apparently no combination with fewer than 96 devices 
could be found. This global best design is pictured in Fig. 7. The design had three arrays on the upper wing surface and two on the lower surface for a total of 96 devices in 5 arrays as compared to 82 devices in 4 arrays selected by the manual method. However, this 96-device design met all the targets while those selected by the manual method did not.

Based on encouraging results from phase I, the GA was used to select individual devices from a set of 349 . This set contained all of the devices that made up the 34 arrays in phase I, plus some other devices that seemed promising.

The phase II GA was tested with a variety of crossover strategies and mutation rates. A typical execution of the phase II GA requires about 8 hours of CPI time. The choice of strategy does not seem to affect the results or the efficiency very much. The results in Fig. 8 were produced with a single-point crossover and a mutation rate of $1 \%$.

The members of the phase II GA population were initially assigned random numbers from 0 to 349 . If the same random number was generated more than once in any member, then all the duplicates were set to zero. Duplicates were similarly set to zero following crossover and mutation operations. The string length was set to 100 , so the number of possible combinations of 100 devices chosen from 349 was $\left(\begin{array}{l}100 \\ 349\end{array}\right) \approx 4 \times 10^{(x)}$.

Because the number of combinations was much larger than in phase I, the population size was increased to 300 and the maximum number of generations was set 10 500 . Thus, about 150.000 individual members were evaluated during each repetition of the GA. This number represented a tiny percentage of all possible combinations.

Higure 8 shows typical convergence performance for the GA, and Fig. 9 shows the 45 devices selected by this execution of the GA. Notice in Fig. 8, that even the "best ever" fitness value was initially above 500 ; this high initial value means that all individuals in the population were heavily penalized. After about 100 generations, the best fitness dropped sharply, indicating that a design meeting the targets had been found.

\section{Simulation of the Control System}

Several effector suites defined with the MATLABbased tool have been applied to the ICE vehicle in a simulation and used in a stability augmentation and control system design (Ref. 9). For the present study, the control system deployed the effectors in a proportional fashion: each device in an effector array was set to the same height. As greater moments were required by the control system, the height of a given effector array was increased until the limiting height was reached. By using these arrays. the control system was able to stabilize and maneuver the vehicle without conventional moving surfaces such as ailerons or a rudder. The predicted authority of these devices was still rather low when compared with that of a rudder or aileron, so the control system generated relatively lowrate maneuvers (roll rates of 5 to 10 degrees per second). Future research will focus on experimental validation of the predicted authority of various flow control devices and on better estimates of their effectiveness.

Figure 10 contains time history plots comparing the phase I (iA suite (thick solid). the original manuallyselected suite (thin dashed), and the ideal moment targets (thin solid). The roll and yaw moments coming from the GA suite look very good-slightly better than those from the original suite. Both suites cause an undesired pitch perturbation (sec Fig. $10 \mathrm{~b}$ ), but the GA results in smaller pitch transients during the maneuver. The crosswind gust capability of the GA effector suite was about the same as the original; the GA suite could tolerate $29.5 \mathrm{ft} / \mathrm{s}$ crosswind gust, while the original suite could withstand a $28 \mathrm{ft} / \mathrm{s}$ gust. From this analysis, the phase I GA appears to have found a good solution. Further testing is required to evaluate the phase II GA solution.

\section{Concluding Remarks}

This paper summarizes several years of research supported by the Aircraft Morphing Program at NASA I angley Research Center. The paper emphasizes the use of MDO techniques applied to the control system design process for novel aircraft configurations. One such novel aircraft concept is the Lockheed-Martin ICE configuration with distributed shape-change devices to generate control moments. Starting with a CFD model of the ICE configuration, this paper shows how the control moments were estimated, promising effector locations were proposed, optimal subsets of these proposed locations were chosen and the control system was simulated and tested.

Obviously, the advanced flow control research is not complete. The concepts must be tested in the wind tunnel, and more effective shape-change devices must be sought. Moreover, both CFD predictions and flight control simulations need to be improved and validated. These improved prediction capabilities may influence the fitness function used for optimization as well as the 
implementation details used for automatic differentiation.

Whilc acknowledging that flow control research is in its infancy, this paper demonstrates useful MDO techniques that will be available to the control designers of the future. The automatic differentiation techniques demonstrated with the ICE model and PMARC code are easily adaptable to other CFD models and codes. Likewise, the genetic algorithms developed herein can be used with improved controleffectiveness measures to find the best locations for a wide variety of shape-change effectors. Thus, simulation techniques such as CFD, as well as MDO techniques such as GA and automatic differentiation, empower engineers to explore revolutionary control concepts for aircraft of the future.

\section{References}

1. Raymer, Daniel P., Aircraft Design: $A$ Conceptual Approach, AIAA, Washington, DC, 1992.

2. Wlezien, R. W., Horner, G. C., McGowan, A. R. Padula, S. L., Scott, M. A., Silcox, R. J., and Simpson, J. O., "The Aircraft Morphing Program," AIAA Paper 99-1927, Apr. 1998.

3. Scott, M., Montgomery, R., and Weston, R., "Subsonic Maneuvering Effectiveness of High Performance Aircraft Which Employ Quasi-Static Shape Change Devices," SPIE 1998 International Symposium on Smart Structures and Materials, Paper 3326-24, pp. 223-233.

4. Dorsett, K. M., and Mehl, D. R., "Innovative Control Effectors (ICE)," Wright Laboratory Report, WL-TR-96-3043, Jan. 1996.

5. Ashby, D., Dudley, M., Iguchi, S., Browne, L., and Katz, J., "Potential Flow Theory and Operation Guide for the Panel Code PMARC_12," NASA Ames Research Center, Moffett Field, CA, Dec. 1992.

6. Carle, A., Fagan, M., and Green, L., "Preliminary Results from the Application of Automated Adjoint Code Generation to CFL3D," AIAA Paper 98-48078, Sept. 1998.

1. Park, M., Green, L.. Montgomery, R., Raney, D., "Determination of Stability and Control Derivatives using Computational Fluid Dynamics and Automatic Differentiation," AIAA Paper 99-3136, June 1999.

8. Carle, A., and Fagan, M., "Overview of ADIFOR-3.0," CAAM-TR $00-02$, Rice
University, Department of Computational and Applied Mathematics, Jan. 2000.

9. Raney, David L., Montgomery, Raymond C., Park, Michael A., and Green, Lawrence L., "Flight Control Using Distributed Shape-Change Effector Arrays," AIAA Paper 2000-1560, Apr. 2000.

10. Padula, Sharon L., and Kincaid, Rex K.: "Optimization Strategies for Sensor and Actuator Placement," NASA TM-1999-209126, Apr. 1999.

1. Rogers, James L., "Optimum Actuator Selection with a Genetic Algorithm for Aircraft Control," Intelligent Engineering Systems Through Artificial Neural Networks, Vol. 9, edited by Dagli, Buczak, Ghosh, Embrechts, and Ersoy, ASME Press, New York, 1999, pp. 355-360.

12. Cook, A., and Crossley, W., "Genetic Algorithm Approaches to Smart Actuator Placement for Aircraft Flight Control," AlAA Paper 2000-1582, Apr. 2000.

13. Goldberg, D., Genetic Algorithms in Search. Optimization and Machine Learning, AddisonWesley Publishing Co., New York, 1989. 
Figures

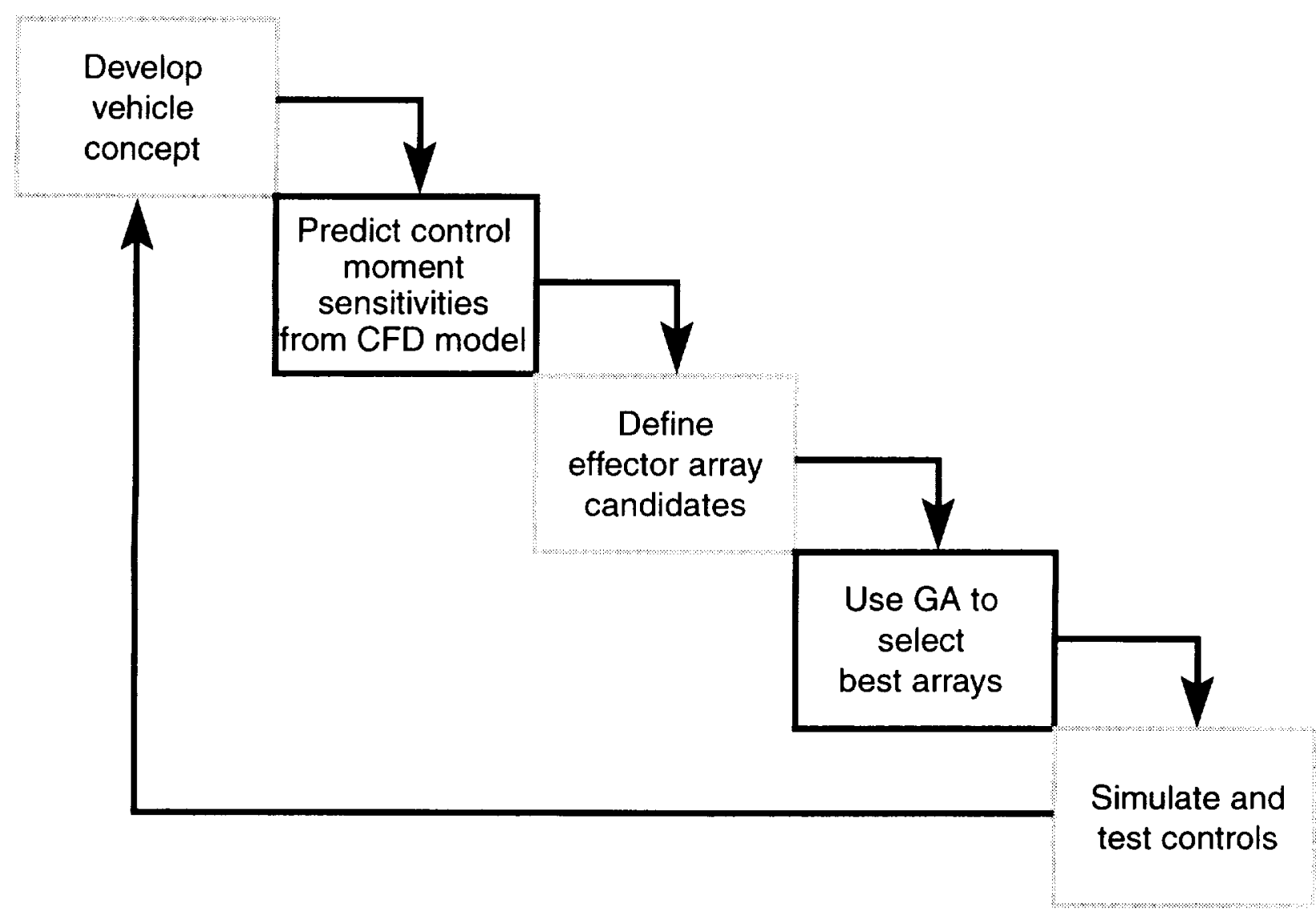

Fig. 1 Control system design process. 

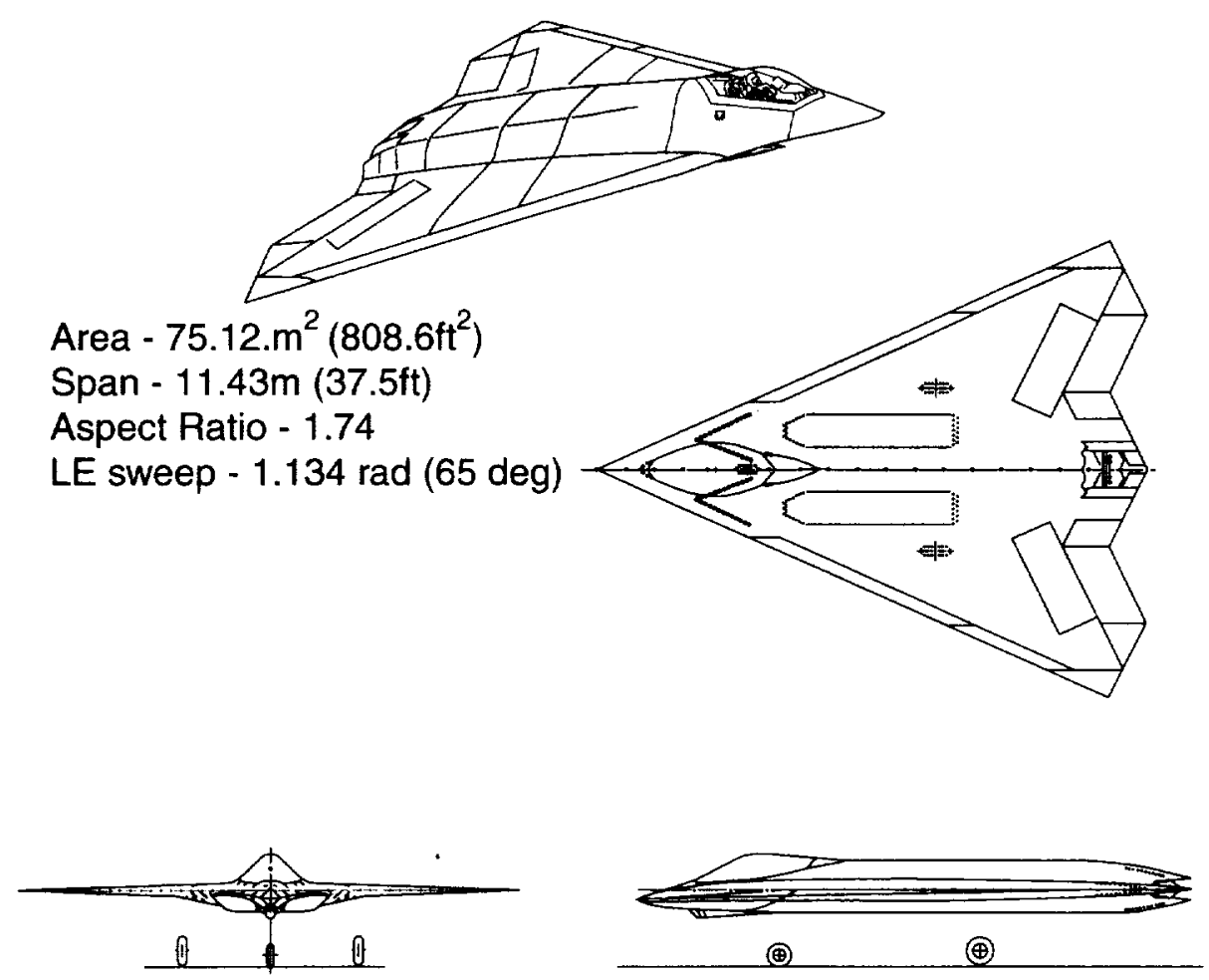

Fig. 2 Lockheed-Martin Innovative Control Effector (ICE) configuration.



Fig. 3 Effector array design tool graphical user interface. 


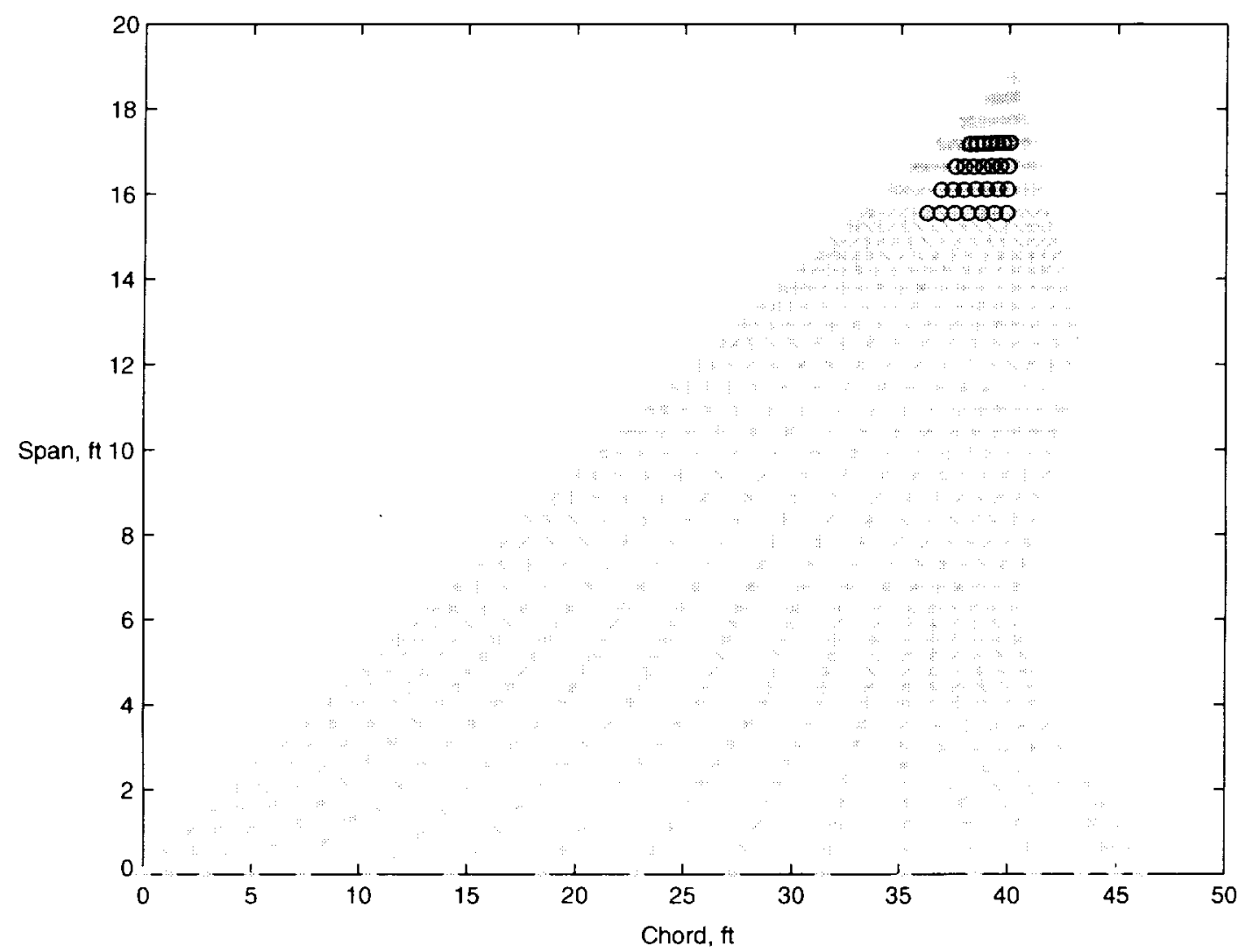

Fig. 4 One of the 34 candidate effector arrays on the right wing upper surface.



Fig. 5 Control authority plots for candidate effector arrays: 7 regions. 


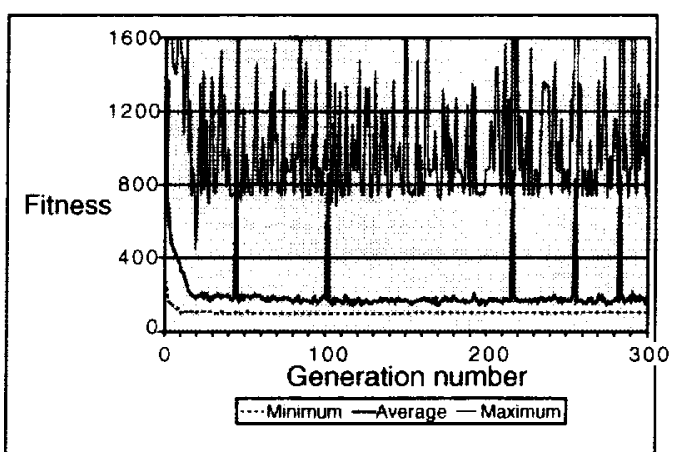

(a) Population extremes

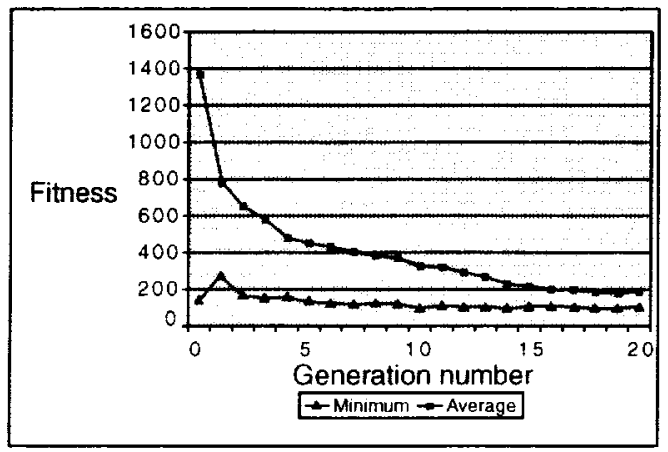

(b) Minimum and average

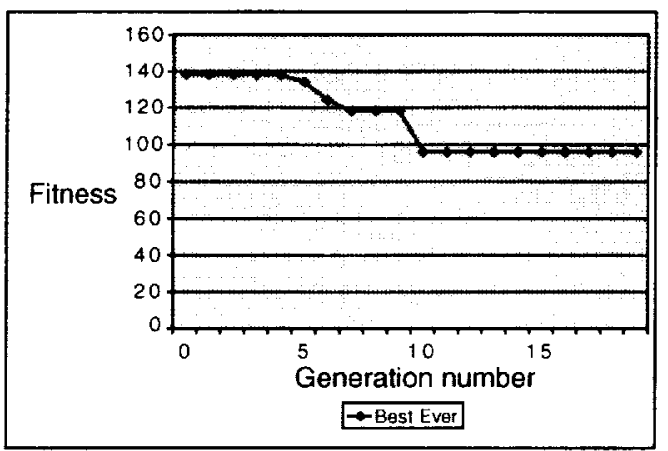

(c) Global best

Fig. 6 Genetic algorithm convergence history.

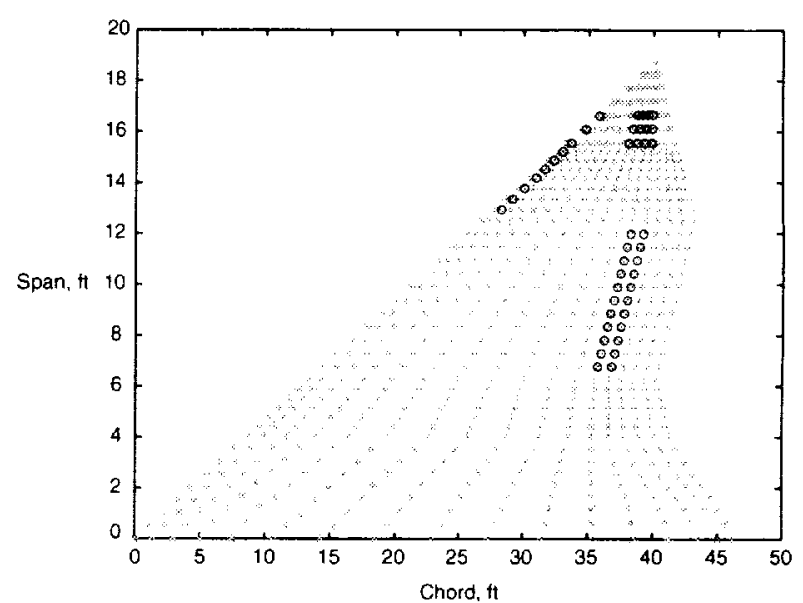

(a) Upper surface arrays

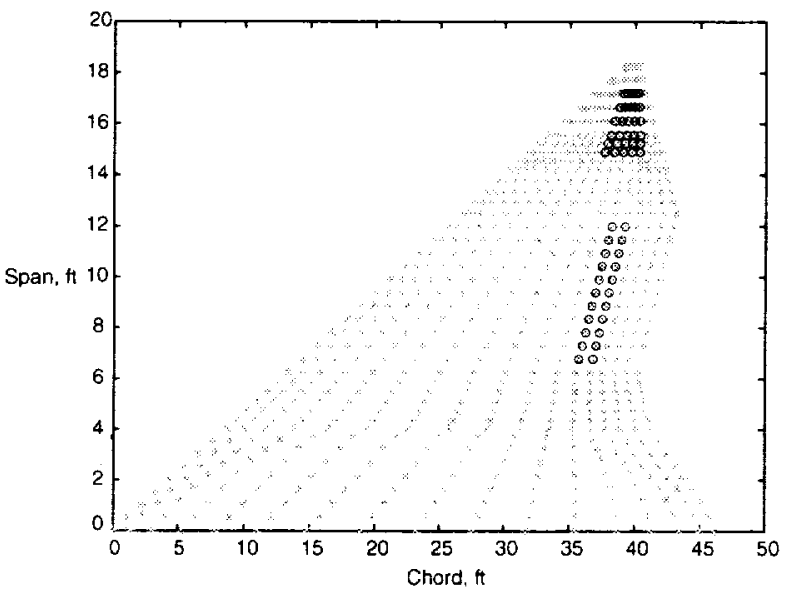

(b) Lower surface arrays

Fig. 7 Best effector suite found by phase I GA.



Fig. 8 Genetic algorithm convergence history. 


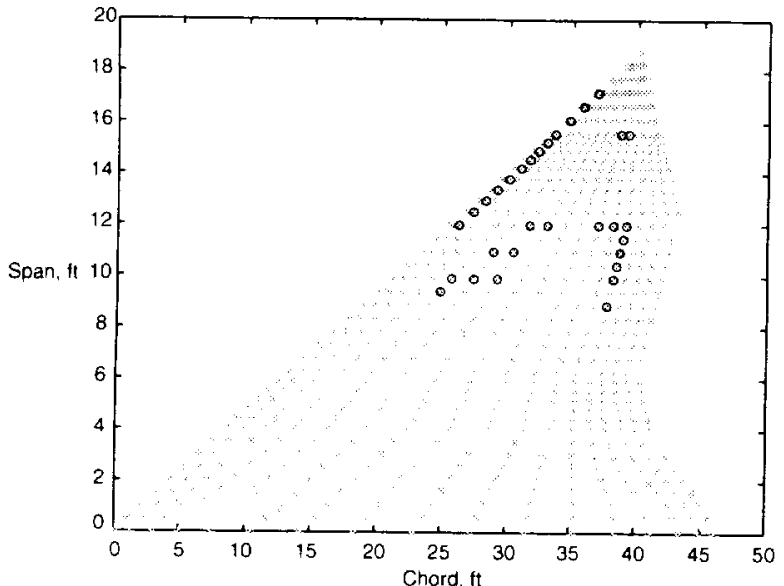

(a) Ilpper surface devices

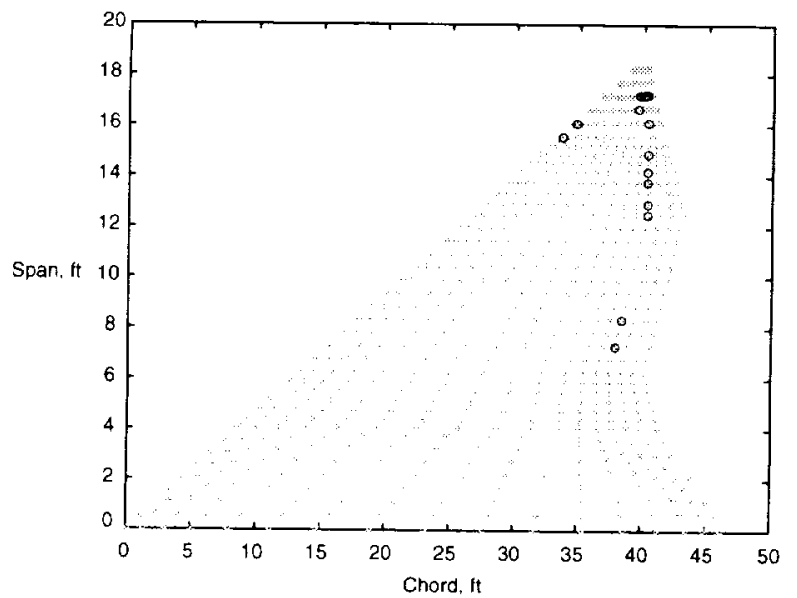

(b) Lower surface devices

Fig. 9 Best set of devices found by phase II GA.

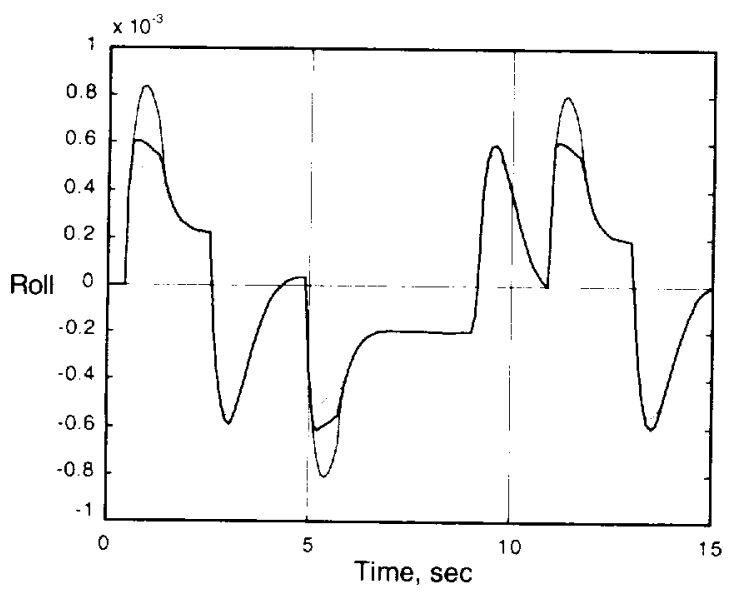

(a) Roll moment time histories

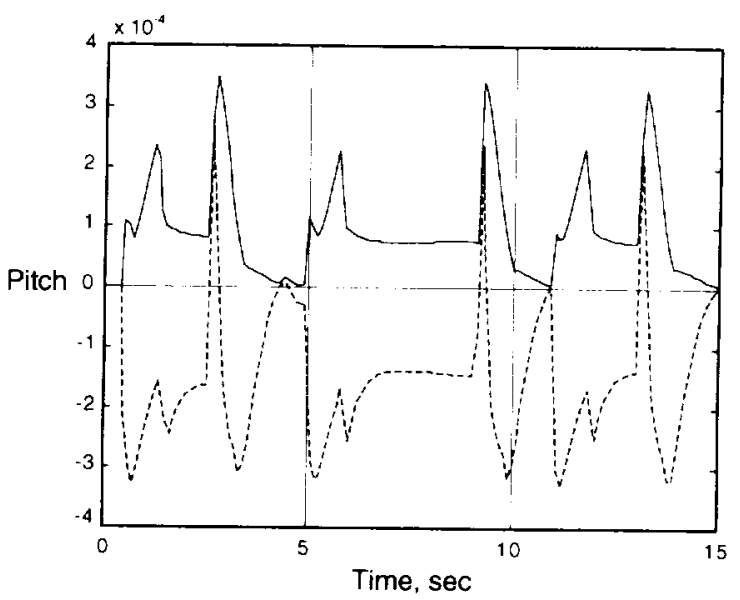

(b) Pitch moment time histories

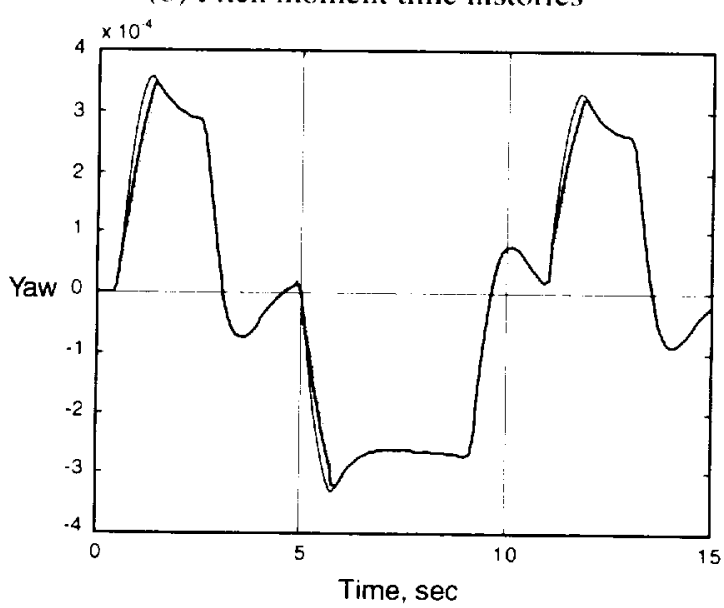

(c) Yaw moment time histories

Fig. 10 Simulation time histories generated in response to a $+1-20$-deg bank angle doublet command. (- ideal - Phase I (iA ...... Ref. 9) 
